\title{
Spatial Interaction of Crime Incidents in Japan ${ }^{1}$
}

\author{
${ }^{1}$ Kazuhiko Kakamu, ${ }^{2}$ Wolfgang Polasek and ${ }^{3}$ Hajime Wago \\ ${ }^{1}$ Department of Economics \& Finance, Institute for Advanced Studies, Stumpergasse 56, 1060, Wien, Austria, \\ ${ }^{2}$ Department of Economics \& Finance, Institute for Advanced Studies, Stumpergasse 56, 1060, Wien, Austria, \\ ${ }^{3}$ Graduate School of Economics, Nagoya University, Chikusa, Nagoya, 464-8601, Japan.
}

Keywords: Crime Incidents, Spatial Autoregressive Model, Panel Data, Markov chain Monte Carlo (MCMC)

\section{EXTENDED ABSTRACT}

Since the seminal work of Becker (1968), a large empirical literature has developed around the estimation and testing of economic models of crime. Recently, spatial interactions of crime incidents have become one of the research areas in economics in connection with the progress of spatial econometrics. But these models of crime based on aggregated data relied heavily on cross-sectional econometric techniques, and therefore previous attempts do not control for unobserved heterogeneity.

Cornwell and Trumbull (1994) show that using panel data gives us a large model class for criminal data. Although there also exist several works on spatial panel models, homogeneity of variance across time is assumed in these models. But with the progress of Bayesian techniques such a model with heteroscedastic variances can be estimated. For example, LeSage (1997) proposes the Bayesian estimation of spatial autoregressive model with heteroscedasticity. In this paper, we extend the approach by LeSage (1997) to spatial Bayesian panel models, using a spatio-temporal heteroscedasticity approach, and examine Japanese criminal time series data. As explanatory variables we use income, the unemployment rate, the number of registered foreigners and the police force. We also use the arrest rate there, since the occurrence of crimes might be related to the apprehension rate. Additionally, the relationship to tourism is modelled by a proxy variable counting hotel nights.

We estimate the model via the Markov chain Monte Carlo (MCMC) method i.e. we sample sequentially through the complete set of full conditional distributions of the parameters. To implement the MCMC sampling approach we need to derive the complete conditional distributions for all parameters in the model. This procedure produces a set of estimates that converges in the limit to the joint posterior distribution of the parameters.

We analyze the development of 18 types of criminal records in Japan for the period 1990 to 2001

\footnotetext{
${ }^{1}$ The research for this work was supported by Grants-In-Aid for Scientific Research (A)(1) 15200022.
}

across 47 prefectures with spatial lag and spatiotemporal heteroscedasticity. We explore the spatial interaction of crime incidents and the hypothesis that crime data are related to socio-demographic variables in Japan. We extend the Bayesian approach of LeSage (1997) for spatio-temporal Bayesian models. Additionally we analyze unobserved heterogeneity and heteroskedasticity in the panel model by variance factors as in Geweke (1993). We can find four major tendencies: (1) We cannot confirm the spatial interaction for any type of crime incidents. (2) The criminal rate becomes high when the Gross Regional Product (GRP) per capita, the unemployment rate and the arrest rate are low (across prefectures) and increases with the number of foreigners (per capita), the police force (per capita) and the number of hotels (per capita). (3) The relative variance of metropolitan areas like Tokyo and Osaka is much larger than in other prefectures. And finally (4) The relative variances increase over time.

Finally, some open issues remain: In this paper, we concentrated on the estimation of spatio-temporal models with heteroskedasticity by an univariate approach. But there may exist the possibility that unobservable factors -not covered by our set of variables-could influence the estimation results. We think that the one of reason that the $R^{2}$ is low comes from the choice of explanatory variables. In addition, as future applications, we also want to consider correlations among crimes, because some kinds of crimes could happen simultaneously. Thus, a more elaborate version of these spatio-temporal panel models could include Seemingly Unrelated Regression (SUR) models. Nevertheless, for the Japanese prefectures our modelling results for 18 crime rates represent a promising first step for more elaborate econometric models to understand the socioeconomic background of crimes.

The paper is organized as follows. In the next section, we introduce our heteroscedastic panel model to examine the spatial interaction of crime incidents and the prior distributions to implement Bayesian estimation. Section 3 derives the conditional distributions for our model. Section 4 presents the empirical results based on 18 types of criminal records in Japan from 1990 to 2001. Section 5 summarizes the results with concluding remarks. 


\section{INTRODUCTION}

Since the seminal work of Becker (1968), a large empirical literature has developed around the estimation and testing of economic models of crime. Recently, spatial interactions of crime incidents have become one of the research areas in economics in connection with the progress of spatial econometrics (see e.g., Anselin (1988)). But these models of crime based on aggregated data relied heavily on cross-sectional econometric techniques, and therefore previous attempts do not control for unobserved heterogeneity.

Cornwell and Trumbull (1994) show that using panel data gives us a large model class for criminal data ${ }^{1}$. Although there also exist several works on spatial panel models, e.g., Elhorst (2003), homogeneity of variance across time is assumed in these models. But with the progress of Bayesian techniques such a model with heteroscedastic variances can be estimated. For example, LeSage (1997) proposes the Bayesian estimation of spatial autoregressive model with heteroscedasticity. In this paper, we extend the approach by LeSage (1997) to spatial Bayesian panel models, using a spatio-temporal heteroscedasticity approach, and examine 18 types of criminal time series in Japan for the period 1990 to 2001 across 47 prefectures. As explanatory variables we use income, the unemployment rate, the number of registered foreigners and the police force. We also use the arrest rate there, since the occurrence of crimes might be related to the apprehension rate. Additionally, the relationship to tourism is modelled by a proxy variable counting hotel nights.

From our empirical results, we can find four major tendencies: (1) We cannot confirm that the spatial interaction for any type of crime incidents is different from previous studies e.g., Anselin (1988) and so on. (2) The criminal rate becomes high when the Gross Regional Product (GRP) per capita, the unemployment rate and the arrest rate are low (across prefectures) and increases with the number of foreigners (per capita), the police force (per capita) and the number of hotels (per capita). (3) The relative variance of metropolitan areas like Tokyo and Osaka is much larger than in other prefectures. And finally (4) The relative variances increase over time.

This paper is organized as follows. In the next section, we introduce our heteroscedastic panel model to examine the spatial interaction of crime incidents and the prior distributions to implement Bayesian estimation. Section 3 derives the conditional

\footnotetext{
${ }^{1}$ The Cornwell and Trumbull's (1994) approach is a little bit different from our approach in so far as they also focus on simultaneity. But it is notable that they address the fact that the panel data gives us a large modeling variety for criminal models.
}

distributions for our model. Section 4 presents the empirical results based on 18 types of criminal records in Japan from 1990 to 2001. Section 5 summarizes the results with concluding remarks.

\section{MODEL}

We have introduced spatial interaction terms in our model and we consider the fixed effect model with spatial lags and spatio-temporal heteroscedasticity.

Suppose $Y=\left(Y_{1}^{\prime}, \cdots, Y_{t}^{\prime}, \cdots, Y_{T}^{\prime}\right)^{\prime}$ denote a dependent variable where $Y_{t}=\left(Y_{1 t}, \cdots, Y_{i t}, \cdots, Y_{N t}\right)^{\prime}$ and $X=\left(X_{1}^{\prime}, \cdots, X_{t}^{\prime}, \cdots, X_{T}^{\prime}\right)^{\prime}$ denote a set of $k$ independent variables where $X_{t}=$ $\left(X_{1 t}^{\prime}, \cdots, X_{i t}^{\prime}, \cdots, X_{N t}^{\prime}\right)^{\prime} . \quad i(=1, \cdots, N)$ refers to a spatial unit, $t(=1, \cdots, T)$ refers to a given time period and $\beta=\left(\beta_{1}, \cdots, \beta_{k}\right)^{\prime}$ are fixed but unknown parameters. Then, the fixed effect model with spatial lag and spatio-temporal heteroscedasticity is represented as follows;

$$
\begin{aligned}
Y_{t} & =\rho W Y_{t}+X_{t} \beta+\mu+\epsilon_{t} \\
\epsilon_{t} & \sim N\left(0, \sigma^{2} V_{t}\right) \\
V_{t} & =\operatorname{diag}\left(v_{1 t}, v_{2 t}, \cdots, v_{N t}\right)
\end{aligned}
$$

where $\epsilon_{t}=\left(\epsilon_{1 t}, \cdots, \epsilon_{N t}\right)^{\prime}$ and $\mu=\left(\mu_{1}, \cdots, \mu_{N}\right)^{\prime}$. $V_{t}$ is unknown diagonal matrix and $\mu_{i}$ are the effect of the omitted variables that are specific to each spatial unit. $W$ is the known $N \times N$ spatial weight matrix, describing the first-order contiguity relationship (it can be also a function of distance). It is assumed that $W$ is a weight matrix of known constants, with all diagonal elements set to zero, and that it is row stochastic matrix; that is, the row sum is $1: W \iota=\iota$, where $\iota$ denotes a vector of ones. Then, the likelihood function of this model is given as

$$
\begin{aligned}
L\left(Y \mid \sigma^{2}, \beta, \rho, X, W\right) & \\
& =\frac{1}{{\sqrt{2 \pi \sigma^{2}}}^{N \cdot T}}\left|I_{n}-\rho W\right|^{T}|V|^{-1 / 2} \\
& \exp \left(-\frac{1}{2 \sigma^{2}}\left(e^{\prime} V^{-1} e\right)\right),
\end{aligned}
$$

where $e=\left(e_{1}^{\prime}, e_{2}^{\prime}, \cdots, e_{T}^{\prime}\right)^{\prime}, e_{t}=\left(I_{n}-\rho W\right)\left(Y_{t}-\right.$ $\bar{Y})-\left(X_{t}-\bar{X}\right) \beta, \bar{Y}=\left(\bar{Y}_{1}, \cdots, \bar{Y}_{i}, \cdots \bar{Y}_{N}\right)^{\prime}, \bar{X}=$ $\left(\bar{X}_{1}^{\prime}, \cdots, \bar{X}_{i}^{\prime}, \cdots \bar{X}_{N}^{\prime}\right)^{\prime}, V=\operatorname{diag}\left(V_{1}, V_{2}, \cdots, V_{T}\right)$, $\bar{Y}_{i}$ and $\bar{X}_{i}^{\prime}$ means the average of $i$ th unit through time 1 to $T$ in each variables and $I_{n}$ denotes the $N \times N$ identity matrix.

While this model could be estimated using the maximum likelihood method like e.g. in Elhorst (2003), if $V$ is known, the following Bayesian approach allows a numerical analysis of the joint parameter distribution including the estimation of the variance factors $v_{i t}(i=1, \cdots, N, t=1, \cdots, T)$ by simulating the posterior distribution of the model. 
Assume that $\pi\left(\sigma^{2}, \beta, V, \rho\right)=\pi\left(\sigma^{2}\right) \pi(\beta) \pi(V) \pi(\rho)$; that is, the prior parameter distributions are independent. Following Geweke (1993), independent priors $\pi\left(v_{i t}\right)$ are also assigned for all of the relative variance parameters $v_{i t}, i=1, \cdots, N$ and $t=1, \cdots, T$. This leads to the distribution

$$
\begin{gathered}
\pi\left(v_{i t} \mid q_{*}\right)=\left(q_{*} / 2\right)^{q_{*} / 2}\left[\Gamma\left(q_{*} / 2\right)\right]^{-1} v_{i t}^{-\left(q_{*}+2\right) / 2} \\
\exp \left(-q_{*} / 2 v_{i t}\right)
\end{gathered}
$$

which implies that $\pi\left(v_{i t}^{-1} \mid q_{*}\right) \sim \chi^{2}\left(q_{*}\right) / q$. Next, the hierarchical prior distribution is specified as

$$
\begin{aligned}
\pi\left(\sigma^{2}\right) & \sim G a^{-1}\left(N T, s_{*}^{2}\right) \\
\pi(\beta) \sim & N(\beta, H) \text { with } \beta=0, H^{-1}=I_{n} / 1000 \\
\pi\left(v_{i t}^{-1} \mid q_{*}\right) \sim \chi^{2}\left(q_{*}\right) / q_{*} & (\text { iid for } i=1, \cdots, N, t=1, \cdots, T) \\
\pi(\rho) & \sim \text { Unif }(-1,1)
\end{aligned}
$$

The prior hyper-parameters $s_{*}^{2}$ are specified in advance, e.g. $q_{*}=5$ and $s_{*}^{2}$ close to zero. Then the joint posterior distribution of the parameters is given by

$$
\begin{aligned}
p\left(\sigma^{2}, \beta,\right. & \left.V_{n}, \rho \mid Y, X, W\right) \\
\propto & L\left(Y \mid \sigma^{2}, \beta, \rho, X, W\right) \pi\left(\sigma^{2}\right) \pi(\beta) \pi\left(V_{n}\right) \pi(\rho) \\
\propto & \sigma^{-(N \cdot T+1)}\left|I_{n}-\rho W\right|^{T} \\
& \prod_{t=1}^{T} \prod_{i=1}^{N} v_{i t}^{-\left(q_{*}+3\right) / 2} \exp \left(-q_{*} / 2 v_{i t}\right) \\
& \exp \left(-\sigma^{-2} e_{i t}^{2} / 2 v_{i t}\right)
\end{aligned}
$$

where $e_{i t}$ is the $i$ th element of the residual vector $e_{t}=$ $\left(Y_{t}-\rho W Y_{t}-X_{t} \beta\right)$. This density is used to derive the full conditional posterior distributions (fcd's) for all the parameters in the model.

\section{ESTIMATING THE MODEL}

We estimate the model via the Markov chain Monte Carlo (MCMC) method i.e. we sample sequentially through the complete set of full conditional distributions of the parameters. To implement the MCMC sampling approach we need to derive the complete conditional distributions for all parameters in the model. This procedure produces a set of estimates that converges in the limit to the joint posterior distribution of the parameters (see Gelfand and Smith (1990)).

The full conditional posterior distributions for each parameter are described in section 3. Details of the sampling algorithm are given in section 3.5. (see also Tierny (1994)).

\subsection{The Full Conditional Posterior Distribution} for $\sigma^{2}$

From the joint distribution (1) it follows that $p(\sigma \mid \beta, V, \rho) \propto \sigma^{-(N \cdot T+1)} \exp \left(-\frac{1}{2 \sigma^{2}}\left(e^{\prime} V^{-1} e\right)\right)$,

i.e. it follows the inverse chi distribution with scale parameter $\frac{N \cdot T}{2}$ and $\frac{e^{\prime} V^{-1} e}{2}$ degrees of freedom. Therefore, $\sigma^{2}$ follows the inverse gamma distribution, $G a^{-1}\left(\frac{N \cdot T}{2}, \frac{e^{\prime} V^{-1} e}{2}\right)$.

\subsection{The Full Conditional Posterior Distribution for $\beta$}

From the joint distribution (1) the kernel of the distribution of the regression parameter is given by

$$
p(\beta \mid \sigma, V, \rho) \propto \exp \left(-\frac{1}{2 \sigma^{2}}\left(e^{\prime} V^{-1} e\right)\right) .
$$

Thus, the parameter vector $\beta$ conditional on the other parameters in the model, $\sigma^{2}, V, \rho$, is normally distributed:

$$
\begin{aligned}
& p(\beta \mid \sigma, V, \rho) \sim N\left(\hat{\beta}, \sigma^{2} H\right), \\
& \hat{\beta}=\left(X^{\prime} V^{-1} X\right)^{-1} X^{\prime} V^{-1}\left(I_{T} \otimes\left(I_{n}-\rho W\right)\right) Y, \\
& H=\left(X^{\prime} V^{-1} X\right)^{-1},
\end{aligned}
$$

where $I_{T}$ and $\otimes$ denote the $T \times T$ identity matrix and the Kronecker product, respectively.Note that given the parameters $\sigma, V$ and $\rho$, the vector $\left(I_{T} \otimes\right.$ $\left.\left(I_{n}-\rho W\right)\right) Y$ and $X^{\prime} V^{-1} X$ can be treated as known, making this conditional distribution easy to compute and to sample from. This is often the case in MCMC estimation, which makes the method attractive.

\subsection{The Full Conditional Posterior Distribution for $V$}

From the joint distribution (1) we find that

$$
\begin{aligned}
p(V \mid \sigma, \beta, \rho) \propto & \prod_{t=1}^{T} \prod_{i=1}^{N} v_{i t}^{-\left(q_{*}+3\right) / 2} \exp \left(-q_{*} / 2 v_{i t}\right) \\
& \exp \left(-\sigma^{-2} e_{i t}^{2} / 2 v_{i t}\right),
\end{aligned}
$$

Following Geweke (1993), the posterior distribution of the elements of $V$, i.e. $v_{11}, v_{21}, \cdots, v_{i t}, \cdots, v_{N T}$, (conditional on $\sigma, \beta$ and $\rho$ ) is chi-squared distributed

$$
\begin{aligned}
\left(\sigma^{-2} e_{i t}^{2}+q_{*}\right) / v_{i t} \sim \chi^{2}\left(q_{*}+1\right), & \\
& i=1, \cdots, N, t=1, \cdots, T .
\end{aligned}
$$

The (positive) hyper-parameter $q_{*}$ controls the amount of dispersion in the $v_{i t}$ across observations. Alternative values for this parameter produces different families of prior densities $v_{i t}$, with small values of $q_{*}$ producing leptokurtic distributions and large values $\left(q_{*} \rightarrow \infty\right)$ imply homoscedasticity. 


\subsection{The Full Conditional Posterior Distribution for $\rho$}

From the joint distribution (1) the spatial correlation parameter follows the kernel

$p(\rho \mid \sigma, \beta, V) \propto\left|I_{n}-\rho W\right|^{T} \exp \left(-\frac{1}{2 \sigma^{2}}\left(e^{\prime} V^{-1} e\right)\right)$.

As is $\rho$ is contained in the determinant, it is difficult to sample from the conditional posterior distribution. Therefore, we adopt a Metropolis step with rejection sampling.

\subsection{The MCMC Sampler}

The MCMC estimation scheme can be started with arbitrary initial values for the parameters which are denoted by $\sigma^{0}, \beta^{0}, V^{0}$ and $\rho^{0}$. Then we sample sequentially from the following set of conditional distributions for the parameters:

1. $p\left(\sigma \mid \beta^{0}, V^{0}, \rho^{0}\right) \sim G a^{-\frac{1}{2}}\left(\frac{N \cdot T}{2}, \frac{e^{\prime} V^{-1} e}{2}\right)$. This new drawn value for the parameter $\sigma$ we label $\sigma^{1}$.

2. $p\left(\beta \mid \sigma^{1}, V^{0}, \rho^{0}\right)$, which we sample from multivariate normal distribution in (2) with mean $\hat{\beta}$ and variance $\sigma^{2} H$. We label these new drawn parameters $\beta^{1}$.

3. $p\left(v_{i t} \mid \sigma^{1}, \beta^{1}, v_{-i t}, \rho^{0}\right)$, which is chi-square distributed with $q+1$ degrees of freedom as shown in (3), where $v_{-i t}=$ $\left(v_{11}, v_{21}, \cdots, v_{i-1 t}, v_{i+1 t}, \cdots, v_{N T}\right)$.

4. The following Metropolis step is used: Sample $\rho$ from

$$
\rho=\rho^{0}+c \theta, \theta \sim N(0,1)
$$

where $c$ is called tuning parameter ${ }^{2}$. Next, we evaluate the acceptance probability

$$
\alpha\left(\rho^{0}, \rho\right)=\min \left(\frac{p(\rho)}{p\left(\rho^{0}\right)}, 1\right),
$$

and finally set $\rho^{1}=\rho$ with probability $\alpha\left(\rho^{0}, \rho\right)$, otherwise $\rho^{1}=\rho^{0}$. If $\lambda_{\min }$ and $\lambda_{\max }$ denote the minimum and maximum eigenvalues of $W$, it is well known that $\lambda_{\min }<0$ and $\lambda_{\max }>0$ and $\rho$ must lie in the interval, $\rho \in\left[\lambda_{\min }^{-1}, \lambda_{\max }^{-1}\right]$ as is shown in Sun et al.(1999). Therefore, we adopt the rejection sampling to constrain $\rho$ to the desired interval using the Metropolis algorithm (see Smith and LeSage (2004)).

${ }^{2}$ As is mentioned in Roberts et al. (1997), it is desirable to select $c$ as the acceptance rate becomes about $25 \%$. We choose $c=0.05$ to make the acceptance rate about $25 \%$ in this paper.
We now return to step 1 employing the updated parameter values in place of the initial values $\sigma^{0}$, $\beta^{0}, V^{0}$ and $\rho^{0}$. On each pass through the iteration we collect the parameter draws which are used to construct a posterior distribution for the parameters in the model.

\section{EMPIRICAL RESULTS}

\subsection{Data Base}

First we would like to explain the data set used in this paper. The criminal records are obtained from the (annual report on) Crime Statistics prepared by the National Police Agency of Japan, which reports 18 types of crimes. In this paper, we explore the hypothesis that crime data are related to socio-demographic variables, like e.g. income, unemployment, foreigners and the size of the police force. For income, we use the Gross Regional Product $(G R P)$ from Vital Statistics prepared by the Cabinet Office of Japan, for the unemployment rate (UNEMP) the Basic Survey on Wage Structure prepared by the Ministry of Health, Labour and Welfare of Japan, for the registered foreigners (FOREIGN) we use Statistics on Immigration Control prepared by the Ministry of Justice of Japan and for the number of policemen (POLICE) the Survey on Wages of Local Government Employees prepared by the Ministry of Internal Affairs and Communications of Japan. Crimes might also depend on the arrest rate (ARREST), which is calculated as the number of arrests ARREST by criminal records. Additionally, the relationship to tourism is explored. Since there are only a few statistics available on tourism in Japan, we use the number of hotels (HOTEL) from the Reports of Health Administration prepared by the Ministry of Health, Labour and Welfare of Japan as a proxy variable for tourism. All the data are annual data and obtained for the period 1990 to 2001 across 47 prefectures. Following the previous literature we use standardized (per capita) data: all dependent and independent variables except the UNEMP and ARREST variables are divided by the population in each prefecture (from Vital Statistics of Japan provided by the Ministry of Health, Labour and Welfare).

As the weight matrix $W$ we use the contiguity dummy variables (see Anselin, 1988). All except one (Okinawa) Japanese prefectures are situated on the four major islands, Hokkaido, Honshu, Shikoku and Kyushu. But these four islands are connected by train and roads, despite the fact that islands are separate geographical entities. But for example, the most northern island Hokkaido is connected by the Seikan railway tunnel to Honshu. And Honshu is connected by the Awaji and Seto Bridge to Shikoku, and the southern island of Kyushu is also connected by the Kanmon Tunnel and Bridge to Honshu. 
Therefore, considering such connections we could build a contiguity weight matrix in which Okinawa is the only prefecture which is independent of all other prefectures.

\subsection{Estimation}

We ran the MCMC algorithm for 5000 iterations following a burn-in phase of 1000 iterations. The chain was considered to have practically converged after 1000 iterations based on a diagnostic proposed by Geweke (1992). Table 2 shows the regression estimates for the 18 types of crimes. We have marked the significance of estimated parameters, by indicating if zero is included in the $95 \%$ (posterior) credible interval or not. First of all, we notice that the spatial correlation coefficient is not" significant", i.e. zero is included in all $95 \%$ (posterior) credible intervals of the 18 models. Interestingly, all spatial correlations but 1 (for assembly crimes) are negative. High (or low) crime rates in the neighboring prefecture do not show a spill-over into other prefectures. For example, Anselin (1998) uses only the simple autoregressive model, but our model is more complex because we use panel data with heteroskedastic variance factors.

\subsection{The Socio-Economic Profiles of Crimes}

We found the following tendencies: In general, negative job market conditions and poorer macroeconomic conditions do not lead to more crimes in Japan. High crime rates are also associated with a large police force, but nothing can be said for the causality, except that EMBEZZLE, TRUST and GAMBLING seem to be difficult to be deterred by a large police force. A higher foreigner rate is connected with a higher crime rate. In $50 \%$ of the crime types the variable hotel (and therefore tourism) is connected with a higher crime rate. In both cases it is difficult to say something for the direction of the causality. The results for the arrest rate are very mixed. In 5 types of crimes the arrest rate helps to drive down the crime rate, but in 6 types of crimes (one third of the crime types) the association is positive.

Finally, we have examined the estimated relative variances to study heteroskedasticity effects. Figure 2 shows the relative variance factors' dynamics for 4 types of crimes through 1990 to 2001 as examples. From the figures we can identify the prefectures where the relative variances are large. These are the metropolitan areas of Tokyo and Osaka, together with their neighbors. Note that there exists the possibility that unobservable factors - not covered by our set of variables- could influence the estimation results. We also see from the figures that there exists something like a variance inflation trend over time, i.e. that the relative variances become larger over time except for those prefectures that started out with large relative variances. Also, it could be that unobservable factors become larger over time. These results indicate that we might need to consider more socio-economic variables for explaining crimes in Japanese prefectures.
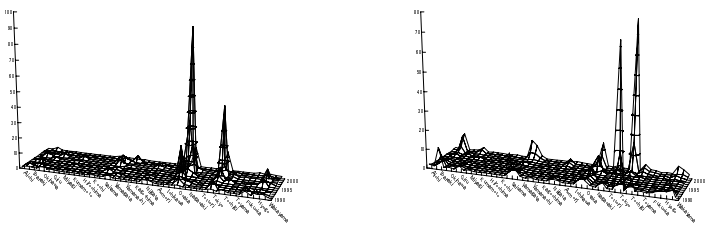

MURDERS ROBBERY
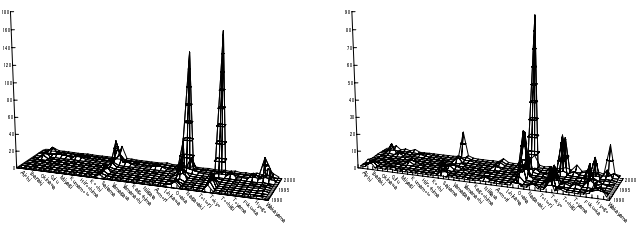

ARSON

$R A P E$

Figure 1. Heteroscedastic Variance Factor

\section{CONCLUDING REMARKS}

We analysed 18 types of crimes in a spatial Bayesian panel model, and found some interesting socioeconomic profiles for crime incidents in Japan. The results indicate that (1) Significant spatial correlation is not present in any of the 18 types of crimes; (2) The crime rate of many crime types correlates negatively with UNEMP, GRP and ARREST and positively with FOREIGN, POLICE and HOTEL; (3) the relative variance of the crime rate in metropolitan areas like Tokyo and Osaka is much larger than in other prefectures and (4) the relative variance becomes larger over time in non-metropolitan areas.

Finally, some open issues remain: In this paper, we concentrated on the estimation of spatio-temporal models with heteroskedasticity by an univariate approach. But there may exist the possibility that unobservable factors - not covered by our set of variables- could influence the estimation results. We think that the one of reason that the $R^{2}$ is low comes from the choice of explanatory variables. In addition, as future applications, we also want to consider correlations among crimes, because some kinds of crimes could happen simultaneously. Thus, a more elaborate version of these spatio-temporal 
panel models could include Seemingly Unrelated Regression (SUR) models. Nevertheless, for the Japanese prefectures our modelling results for 18 crime rates represent a promising first step for more elaborate econometric models to understand the socioeconomic background of crimes.

\section{ACKNOWLEDGEMENTS}

The computational results are obtained using Ox (see Doornik, 2001).

\section{REFERENCES}

Anselin, Luc (1988) Spatial Econometrics: Methods and Models, Dordrecht: Kluwer.

Becker, Gary S. (1968) "Crime and Punishment: An Economic Approach," Journal of Political Economy, 76, 169-217.

Cornwell, Christopher and William N. Trumbull (1994) "Estimating the Economic Model of Crime with Panel Data," Review of Economics and Statistics, 76, 360-366.

Doornik, Jurgen A. (2001) Ox: Object Oriented Matrix Programming, 3.0, Timberlake Consultants Press, London.

Elhorst, Paul J. (2003) "Specification and Estimation of Spatial Panel Data Models," International Regional Science Review, 26, 244-268.

Gelfand, Allan E. and Adrian F. M. Smith (1990) "Sampling-based Approaches to Calculating Marginal Densities," Journal of the American Statistical Association, 85, 398-409.

Geweke, J. (1992) "Evaluating the Accuracy of Sampling-Based Approaches to the Calculation of Posterior Means," in Bayesian Statistics 4, (eds J. M. Bernard, J. O. Berger, A. P. Dawid and A. F. M. Smith), Oxford University Press, Oxford.

Geweke, John (1993) "Bayesian Treatment of the Independent Student- $t$ Linear Model," Journal of Applied Econometrics, 8, 19-40.

LeSage, James P. (1997) "Bayesian Estimation of Spatial Autoregressive Models," International Regional Science Review, 20, 113-129.

Roberts, G. O., A. Gelman and W. R. Gilks (1997) "Weak Convergence and Optimal Scaling of Random Walk Metropolis Algorithm," Annals of Applied Probability, 7, 110-120.

Smith, Tonny E. and James P. LeSage (2004) “A Bayesian Probit Model with Spatial Dependencies," in James P. LeSage and R. Kelley Pace (eds.), Advances in Econometrics: Volume 18: Spatial and Spatiotemporal Econometrics, Elsevier Science, 127-160.
Sun, Dongchu, Robert K. Tsutakawa and Paul L. Speckman (1999) "Posterior Distribution of Hierarchical Models using CAR(1) Distributions," Biometrika, 86, 341-350.

Tierney, Luke (1994) “ Markov Chains for Exploring Posterior Distributions (with discussion)," Annals of Statistics, 22, 1701-1762. 
Table 1. Empirical Results

\begin{tabular}{|c|c|c|c|c|}
\hline & MURDER & ROBBERY & $A R S O N$ & $R A P E$ \\
\hline UNEMP & $\overline{-0.019409}$ & $-3.253784^{*}$ & -0.054559 & $-0.167609^{*}$ \\
\hline$G R P$ & 0.002446 & $-0.093299 *$ & $-0.02254^{*}$ & $-0.01914 *$ \\
\hline POLICE & $0.319037^{*}$ & $41.959406^{*}$ & $0.723155^{*}$ & $1.041594 *$ \\
\hline FREIGN & 0.008965 & $-0.20146^{*}$ & $0.265883^{*}$ & $0.044833 *$ \\
\hline HOTEL & $0.047167 *$ & $5.610594 *$ & $0.740068 *$ & $0.663923 *$ \\
\hline ARREST & -0.001559 & $-0.035291^{*}$ & -0.000978 & $-0.004631 *$ \\
\hline$\rho$ & -0.094884 & -0.319345 & -0.192507 & -0.012878 \\
\hline$\sigma^{2}$ & 0.000003 & 0.003024 & 0.000068 & 0.00002 \\
\hline$R^{2}$ & 0.001159 & 0.094146 & 0.015462 & 0.003384 \\
\hline \multirow[t]{2}{*}{ Acceptance Rate } & 0.398667 & 0.45400 & 0.452833 & 0.420167 \\
\hline & $A S S E M B L Y$ & VIOLENCE & INJURY & THREAT1 \\
\hline UNEMP & $\overline{-0.002103}$ & $-5.487127^{*}$ & $-6.246853^{*}$ & $-0.722797^{*}$ \\
\hline$G R P$ & $0.000273^{*}$ & -0.125186 & $-0.484947^{*}$ & -0.013954 \\
\hline POLICE & $0.037628^{*}$ & $169.808691 *$ & $140.057932 *$ & $20.037478 *$ \\
\hline FREIGN & $-0.002237^{*}$ & 0.172961 & $2.063419 *$ & 0.007531 \\
\hline HOTEL & $-0.015742 *$ & 0.34648 & $7.82711^{*}$ & 0.319702 \\
\hline ARREST & $-0.003447 *$ & 0.029328 & $-0.58045^{*}$ & 0.000171 \\
\hline$\rho$ & 0.053158 & -0.166463 & -0.10684 & -0.286933 \\
\hline$\sigma^{2}$ & 0.000000 & 0.020682 & 0.025783 & 0.000325 \\
\hline$R^{2}$ & 0.004202 & 0.14393 & 0.037301 & 0.16355 \\
\hline \multirow[t]{2}{*}{ Acceptance Rate } & 0.368667 & 0.477667 & 0.438833 & 0.478167 \\
\hline & THREAT2 & THEFT & FRAUD & EMBEZZLE \\
\hline UNEMP & $-5.248931^{*}$ & -241.219631* & 4.841015* & $-0.075494 *$ \\
\hline$G R P$ & $-0.275541^{*}$ & $-12.191975^{*}$ & $0.775502 *$ & $-0.013705^{*}$ \\
\hline POLICE & $137.985763^{*}$ & $6317.108179 *$ & $45.668489 *$ & $-0.743869 *$ \\
\hline FREIGN & $0.576213^{*}$ & $92.473666^{*}$ & $3.854738 *$ & $0.10021 *$ \\
\hline HOTEL & $6.072843^{*}$ & $368.082088 *$ & $-19.497665^{*}$ & $0.459769 *$ \\
\hline ARREST & 0.008086 & -7.547919 & 0.064349 & -0.003443 \\
\hline$\rho$ & -0.351197 & -0.107379 & -0.04907 & -0.015662 \\
\hline$\sigma^{2}$ & 0.015613 & 56.043289 & 0.034241 & 0.000028 \\
\hline$R^{2}$ & 0.04992 & 0.014146 & 0.02657 & 0.006907 \\
\hline \multirow[t]{2}{*}{ Acceptance Rate } & 0.471333 & 0.427167 & 0.461833 & 0.388333 \\
\hline & FORGERY & GRAFT & TRUST & GAMBLING \\
\hline 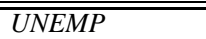 & $2.252524^{*}$ & $-0.018131^{*}$ & $\overline{0.031681^{*}}$ & $\overline{0.429011^{*}}$ \\
\hline$G R P$ & $1.008859^{*}$ & $-0.001439^{*}$ & $-0.002285^{*}$ & $-0.003837^{*}$ \\
\hline POLICE & $74.497379 *$ & 0.039778 & $-0.34323^{*}$ & $-1.772045^{*}$ \\
\hline FREIGN & -0.653028 & $0.0056^{*}$ & $0.019604 *$ & $0.325259 *$ \\
\hline HOTEL & $-24.205143^{*}$ & -0.002505 & 0.012847 & $-0.717944^{*}$ \\
\hline ARREST & 0.024102 & 0.000011 & $-0.000365^{*}$ & -0.001765 \\
\hline$\rho$ & -0.076822 & -0.022695 & -0.201864 & -0.066182 \\
\hline$\sigma^{2}$ & 0.018943 & 0.000001 & 0.000000 & 0.000084 \\
\hline$R^{2}$ & 0.1449 & 0.002857 & 0.11182 & 0.061021 \\
\hline \multirow[t]{2}{*}{ Acceptance Rate } & 0.455333 & 0.391667 & 0.453333 & 0.438333 \\
\hline & OBSCENITY & OTHERS & & \\
\hline UNEMP & $-3.495648^{*}$ & $-38.529497^{*}$ & & \\
\hline$G R P$ & $-0.120715^{*}$ & $-3.678495^{*}$ & & \\
\hline POLICE & $39.444951 *$ & $472.010882 *$ & & \\
\hline FREIGN & $-0.045988^{*}$ & $10.099957 *$ & & \\
\hline HOTEL & $7.02894 *$ & $141.211472 *$ & & \\
\hline ARREST & -0.044106 & -0.454228 & & \\
\hline$\rho$ & -0.162731 & -0.16691 & & \\
\hline$\sigma^{2}$ & 0.003365 & 0.866288 & & \\
\hline$R^{2}$ & 0.094814 & 0.014493 & & \\
\hline Acceptance Rate & 0.446833 & 0.421667 & & \\
\hline
\end{tabular}

Note: * means that the $95 \%$ credible interval does not include zero. 